\title{
Opciones educativas culturalmente sensibles a la diversidad y la implementación de los derechos lingüísticos
}

Educational options culturally sensitive to diversity and the implementation of linguistic rights

Luis Ernesto Behares*

Universidad de la República

Resumo Este trabajo analiza algunas cuestiones teóricas vinculadas a la instrumentalización de los derechos lingüísticos en contextos educativos. En particular, discute las relaciones entre las pedagogías culturalmente sensibles a la diversidad y la instrumentación escolar de derechos lingüísticos de niños y comunidades. Se estudian las tendencias de instrumentación política ligadas a los conceptos de tolerancia, promoción y herencia.

PALAVRAS-CHAVE: Diversidad, Opciones educativas, Derechos lingüísticos.

Abstract This paper analyses some theoretical issues related to the implementation of linguistic rights in educational contexts. In particular, it discusses the relationships between pedagogies that are culturally sensitive towards diversity and the implementation in schools of the linguistic rights of children and communities. This paper studies the tendencies in the implementation of policies tied to the concepts of tolerance, promotion and heritage.

KEYWORDS: Diversity, Educational options, Linguistic rights. 


\section{Comentario inicial}

El avance de las pedagogías sensibles a la diversidad en el ámbito de la educación básica ha permitido alcanzar más certeramente los objetivos, hoy día escasamente discutidos, de inclusión social y cultural de las poblaciones infantiles y adolescentes provenientes de sectores hasta hace poco tiempo excluidos. En una apretadísima síntesis, y con especial atención a nuestros países sudamericanos, podemos a principios de nuestro siglo confirmar la realidad de la instrumentación política y pedagógica de mejores niveles de cobertura, de estrategias de educación y enseñanza técnicamente más ajustadas a las diversidades y la consolidación de una tradición que incorpora los derechos de los participantes en la propia práctica de las instituciones.

Sin embargo, este proceso de consolidación de nuevas tendencias referidas a la atención a las diversidades contiene algunas áreas difusas y siempre deficitarias, no sólo por carencias de financiación, por las particularidades de las acciones políticas fácticas o por la mejor o peor formación de los docentes. En algunas de estas áreas de instrumentación hay, sobre todo, problemas teóricos y jurídicos.

Como se ha señalado insistentemente en los últimos cincuenta años, la atención educativa a la diversidad lingüística y al respeto a los derechos lingüísticos de los participantes (niños, adolescentes y docentes) es uno de los factores principales en todos los niveles de instrumentación que mencionamos, pero también es un área en la que los resultados no son los ideales. En este texto ${ }^{1}$ nos detendremos en el análisis de las indefiniciones teóricas y jurídicas que creemos están en la base de estos logros insatisfactorios.

\section{Los Derechos Lingüísticos}

La noción de "derechos lingüísticos" se articula entre los años de 1945 y 1996, en una serie de documentos de naturaleza variada, pero principalmente correspondientes al ámbito de definición del derecho internacional ${ }^{2}$. En aquellos documentos, la noción se articula en dos direcciones: una de ellas incluye el reconocimiento de lenguas y variedades lingüísticas asociadas a comunidades lingüisticas que conviven con otras en relaciones de poder asimétricas; la otra se refiere a las personas en sus relaciones con las lenguas y el lenguaje (BEHARES, 2013a; 2013b).

Estas tendencias preceptivas comienzan a articularse en el marco de la institución de derechos humanos, sociales y políticos, como correlato de los problemas generados por la segunda guerra mundial y la consolidación del modelo bi-polar en las políticas internacionales. La naciente Organización de las Naciones Unidas (ONU) creyó entonces necesaria la atención a ciertas cuestiones humanitarias, sociales y políticas. En la definición básica de nuevos derechos, operada sobre todo entre 1945 y 1960, se observó, no obstante, la hegemonía de uno de los polos internacionales, a saber el de las naciones adherentes al modelo capitalista-liberal, con notoria directividad de los Estados Unidos. En estas primeras instancias, la atención específica a los derechos lingüísticos es baja.

En términos generales, la atención internacional, regional o nacional al $d e-$ recho en materia lingüistica debió esperar a la década de 1980, pero se constituyó rápidamente en un campo en constante desarrollo, ligado fundamentalmente al derecho 
positivo y tendiente, en general, al establecimiento de políticas lingüísticas. A pesar de esta confirmación, es necesario recordar que la filología, la lingüística, la sociolingüísti$\mathrm{ca} /$ sociología del lenguaje/etnolingüística, las ciencias políticas/jurídicas y la investigación educativa venían desde muchas décadas antes planteando problemas para el campo y produciendo subsidios para su comprensión, sin olvidarnos de las reivindicaciones políticas de las comunidades lingüísticas. En este conjunto de intereses se desarrolló la noción de derechos lingüiśticos, como correlato especializado de la noción de derechos bumanos, con evidentes oscilaciones entre el derecho positivo y el derecho natural ${ }^{3}$.

La noción de derechos humanos es un atributo del pensamiento político de la modernidad. Se articula desde el siglo XVII en la junción del pensamiento liberal con las concepciones medioevales de "derecho natural" (en general, de base teológica), e incluye elementos del pensamiento empirista inglés y del pensamiento racionalismo francés. Se trata, mutatis mutandis, de los derechos de las personas individuales, y de la asunción de que estos derechos son universales y están por encima de cualquier ordenamiento de derecho positivo ${ }^{4}$. La Declaración de la ONU de 1948 se inserta en esta tradición, y hace suyos dos factores nocionales que le son característicos: la afirmación de que los derechos humanos son derechos naturales, y la afirmación de que los derechos humanos descansan y tienen su titularidad exclusivamente en la persona bumana, libre y detentora de una voluntad responsable. Como se observa, se trata del pensamiento liberal, que se asocia a un principio axiomático centrado en la noción de individuo: "La finalidad de toda asociación política es la conservación de los derechos naturales e imprescriptibles del hombre. Esos derechos son la libertad, la propiedad, la seguridad y la resistencia a la opresión" (FRANCIA, 1789, art. II, nuestra traducción).

La Declaración de la ONU de 1948 no hablaba explícitamente de "derechos lingüísticos”. Es el Pacto Internacional de Derechos Civiles y Politicos (ONU, 1966) el que señala por vez primera la inclusión de lo "lingüístico" entre los derechos humanos a tutelar:

En los Estados en que existan minorías étnicas, religiosas o lingüísticas, no se negará a las personas que pertenezcan a dichas minorías el derecho que les corresponde, en común con los demás miembros de su grupo, a tener su propia vida cultural, a profesar y practicar su propia religión y a emplear su propio idioma. (ONU, 1966, art. 27, cursiva nuestra)

Este documento incluye una innovación: el derecho humano universal a su propio idioma en él positivizado no tiene como titular exclusivo al individuo, sino que su titularidad se amplía al "común con los demás miembros de su grupo". De esta forma, la noción de "propio idioma" no se refiere a un derecho exclusivamente individual, cuyo único titular sería la persona. La "propiedad" del idioma, de la cual deriva el derecho, parece descansar más allá de la individualidad y asentarse definidamente en los "grupos" que se identifican como hablantes de un idioma en común.

En los documentos posteriores, más cercanos en el tiempo a nosotros ${ }^{5}$, encontramos este conflicto de titularidad en forma nítida. Ya en la Carta Europea de las lenguas regionales y minoritarias (CMCE, 1992, art. 7, inc. 1), aparece la aserción de que la titularidad para éstos descansa en los colectivos más que en las personas. La Comunidad Europea consagra el derecho de las "lenguas" en sí mismas (usa esta expresión en forma excluyente), como entidades históricas; por lo tanto, la titularidad 
de los derechos que allí se consagran se revierte (al menos en la letra del texto) a los grupos ligados o determinados por las lenguas, y deja de ser de la titularidad de los hablantes como individuos particulares. Luego, en la Declaración Universal de los Derechos Lingüisticos (UNESCO, 1996), documento de naturaleza más "radical" ${ }^{6}$, se reconocen claramente dos tipos de derechos lingüísticos: los individuales y los colectivos. En referencia a los primeros, el argumento sostenido abarca aspectos de orden individual simple (como el derecho al uso público o privado de una lengua), pero enfatiza que los derechos lingüísticos individuales se derivan y se justifican por el derecho lingüístico central de ser reconocido como miembro de una comunidad lingüistica. De esta forma, los derechos colectivos pasan a ser los efectivamente reconocidos, aunque en forma oblicua, y de ellos se desprenderán, por vía de la pertenencia a un colectivo, los derechos individuales. El referente efectivo del goce de los derechos lingüísticos queda en este documento establecido en la comunidad lingüistica histórica:

[...] toda sociedad humana que, asentada históricamente en un espacio territorial determinado, reconocido o no, se autoidentifica como pueblo y ha desarrollado una lengua común como medio de comunicación natural y de cohesión cultural entre sus miembros. La denominación lengua propia de un territorio hace referencia al idioma de la comunidad históricamente establecida en este espacio. (UNESCO, 1996, Art. 1, inc. 1)

La noción de derechos lingüisticos se ha construido como un aspecto especial de los "derechos humanos", pero también se la liga al establecimiento de los ordenamientos sociopolíticos entre los estados o grupos humanos dentro de los estados. La noción incluye, pues, una dimensión referida a los hablantes y otra referida a las comunidades lingüísticas. La noción engloba a los individuos como hablantes y a las comunidades lingüísticas como poseedoras de un bien. Desde la tradición propia de la lingüística podemos hablar de derechos del hablante (o, derechos de lenguaje) y de derechos de las comunidades (o derechos de lengua). La distinción clásica entre lenguaje $y$ lengua establece que el lenguaje es un fenómeno individual, mientras que las lenguas son entidades con un orden propio que está fuera de la individualidad del hablante. Hay, por tanto, un titular individual, en su derecho de hablante, y un titular colectivo socialmente institucional o históricamente determinado en la alteridad de la lengua. Un derecho de hablante se resume, aparentemente, en su derecho a serlo, y por lo tanto a que ningún obstáculo externo tienda a disminuir su acceso espontáneo al ejercicio de lenguaje, mientras que un derecho de lengua se refiere a ésta como entidad histórica reflejada en sus niveles de estabilidad y de "recurrencia", y hace imprescindible la noción concomitante de comunidad lingüística, definible sólo si se la compone como parte de un orden social y político independiente de las voluntades individuales.

En las tradiciones jurídicas es posible oponer derecho natural y derecho positivo. El primero asume la esencialidad de un derecho como atributo inseparable de la persona, en términos absolutos, y por encima de los ordenamientos jurídicos positivos; el segundo es siempre de naturaleza social o política, y consiste en los acuerdos explícitos (positivos) que los ordenamientos jurídicos de los estados o los entes supraestatales proveen. En cierta forma, los derechos del hablante se construyen como derechos lingüisticos naturales integrados a los derechos humanos, mientras que los derechos de lengua o derechos de las comunidades lingüísticas son, por su propia conformación y finalidad, derechos lingüísticos positivos, y lo son porque se los ha establecido en los ordenamientos jurídicos. 


\section{Tolerancia, promoción}

Los parámetros conceptuales y jurídicos que hemos señalado han motivado desde los años de 1990 diversas estrategias de instrumentación en muchos de los Estados y regiones del planeta. Los ámbitos de aplicación de esta nueva dimensión jurídico-lingüística son varios, pero entre ellos se destaca el que se sitúa en los sistemas de educación, como ya mencionamos al inicio de este texto. Este espacio social y político ha sido ya señalado como uno de los que son jerárquicamente más importantes, por su importancia intrínseca y por los efectos de las políticas que a partir de él se derivan. Sin embargo, es también uno de los sectores más conflictivos cuando se pasa a los procesos de instrumentación.

En una apretadísima síntesis, podemos decir que los procesos de instrumentación de los derechos lingüísticos referidos al ámbito educativo, con todas las variaciones propias de los países y sociedades, se han operado en base a tres constructos pragmáticos principales, que se suelen nombrar en la bibliografía como tolerancia, promoción y berencia.

En la terminología acuñada por Kloss (1977), se entiende por tolerancia toda y cualquier política que asegure la no intervención en el derecho individual de las personas en relación a su uso del lenguaje. Es, por lo tanto, una posición de naturaleza pasiva y privada. Por su parte, la promoción es de naturaleza activa y pública, ya que implica acciones para asegurar a las comunidades la utilización de su lengua en los espacios sociales en los que podría utilizarse otra. Si nos referimos al ámbito educativo, se trata de la tolerancia de la diversidad lingüística de los alumnos y de sus docentes y de la promoción de sus lenguas en los sistemas educativos nacionales. Como ha señalado Kloss (1977), las diferencias entre las estrategias de tolerancia y de promoción se derivan del énfasis en la orientación que se les confiere a los derechos. La tolerancia se refiere fundamentalmente a las personas y al ámbito privado de sus relaciones con las lenguas (por ejemplo, el derecho a usar su lengua materna en la vida cotidiana); la promoción, por su parte, va dirigida a las comunidades en la esfera pública (por ejemplo, el reconocimiento legal de lenguas minoritarias y las propuestas para su uso).

El esquema de Kloss (1977) implica cierta posición "benevolente" hacia la persona particular, referida substancialmente al respeto natural de sus derechos humanos, por vía de la omisión de medidas limitantes, en cierto modo compensatoriamente; en cambio, la promoción se articula con mayor dificultad, porque refiere al reconocimiento de derechos positivos de las comunidades o grupos, principalmente los minoritarios. Para la óptica de los organismos internacionales orientados explícitamente en la tradición liberal entre 1948 y 1992, la modalidad política que Kloss llama "promoción" fue claramente desatendida, ya que escapaba a la posibilidad o intención de ser instrumentada en términos reales. Sin embargo, como muestra Varennes (1996), en los años de 1950 es posible situar ya en la jurisprudencia internacional una definición política posible de lengua minoritaria alternativa a la tomado por los organismos internacionales, fundada ésta en el derecho de "propiedad" de un bien colectivo, de naturaleza comunal, establecido o reclamado por una comunidad lingüística particular.

Es necesario recordar aquí que el concepto de derechos bumanos del siglo $\mathrm{XX}$ (generados en los últimos 60 años), y de los derechos lingüísticos dentro de ellos, incorpora la concepción de "derechos" propia del liberalismo político y social anglo- 
sajón, que los restringe casi exclusivamente a la persona individual, por lo cual genera una inhibición para el reconocimiento de bienes colectivos (lenguas u otros) de las comunidades o grupos particulares. La condición de ciudadano en las democracias liberales, según el liberalismo ortodoxo, no incorpora las diferencias de origen grupal y lingüístico ${ }^{7}$, ya que considera a todas las personas como intercambiables y sólo definibles por condiciones generales (YOUNG, 1993).

Las discusiones entre las tendencias individualistas y las posiciones colectivistas (o comunalistas) en esos marcos de referencia ideológicos son abigarradas y se vuelven particularmente críticas en su relación con las opciones educativas. May (2010: p. 134) ha escrito recientemente, al referirse a las críticas comunalistas o colectivistas al liberalismo, que éstas "señalan que la separación estricta de la ciudadanía y la identidad en la política moderna subestima, y niega a veces, el significado de organizaciones comunitarias más extensas, incluyendo la o las lenguas de una persona, en la construcción de la identidad individual". En la sociedad democrática liberal, y en la construcción teórica que la sostiene, los bienes comunes, como las lenguas, sólo pueden ser considerados en relación a los individuos, ya que ambas se sustentan en el individualismo intrínseco. Las políticas referidas a los bienes comunales son entonces tangenciales y aleatorias (VAN DYKE, 1977).

El Artículo 27 del Pacto Internacional de Derechos Civiles y Politicos (ONU, 1966), ya citado en este texto, ha sido para la extensa bibliografía al respecto el articulador político-jurídico principal de estas construcciones que venimos señalando y de los conflictos interpretativos que mencionamos. En él encontramos varios subterfugios:

a) da a los estados la capacidad de decidir si efectivamente tienen o no minorías étnicas, religiosas o lingüísticas;

b) no dice "se dará", sino "no se negará" el derecho a utilizar su propio idioma;

c) la expresión "minorías" fue substituida a lo largo de su discusión por "personas pertenecientes a minorías"; y

d) la opción por minimiza el factor lingüístico comunitario mediante la expresión "en común con los demás miembros de su grupo".

Según varios investigadores (CAPOTORTI, 1979; SKUTNABB-KANGAS, 1998; DE VARENNES, 1996; MAY, 2010), el Artículo 27 refleja la tensión entre los derechos individuales y los derechos de las comunidades y abre una brecha para hacer muy confusa la cuestión de quién puede ejercer o reclamar estos derechos ${ }^{8}$.

\section{La "propiedad" de una lengua, la herencia}

Las políticas de tolerancia y de promoción en las construcciones liberales se enfrentan, como constructos operativos, con la cuestión de la propiedad de una lengua y con las relaciones de ésta con las identidades lingüísticas y las incorporaciones o anexiones de comunidades lingüísticas a los espacios políticos nacionales. Como ha sido evidente para el pensamiento lingüístico, sea en su vertiente funcionalista bloomfieldiana o en la tradición formalista saussureana, la propiedad de una lengua no descansa en el individuo, sino en la comunidad lingüística etnográficamente considerada o en la masa de sus hablantes. Por lo tanto, en este pensamiento ampliamente coherente y especializado, ya desde las formulaciones clásicas de origen filosófico germano en el siglo XVIII, se entiende que una lengua implica algo más que un instrumento de 
comunicación exterior e intercambiable para las personas. Ese algo más no se refiere a la "propiedad" en un sentido jurídico práctico menor o administrable (como cuando hablamos de la propiedad de un bien mueble o inmueble), sino a la historicidad de la relación lengua-comunidad-identidad.

Para ponerlo en términos concretos y a modo de ejemplo: ¿Es Achokalla, que habla aymara como lengua materna en el Paraguay, el dueño de esa lengua? Si aceptamos que lo es, ya que decimos "su lengua", ¿qué sentido de propiedad es el que allí se define? Si no aceptamos que lo sea, ya que para que Achokalla fuera hablante nativo de aymara esta lengua debería haber estado activa antes e independientemente de la existencia de Achokalla, ¿podemos decir que en tanto hablante Achokalla es propiedad de una entidad mayor, sea ésta una comunidad o una etnia? ¿Cuál sería el estatuto jurídico posible de esa relación de propiedad hereditaria entre la etnia aymara y Achokalla como hablante? Considerando que Achokalla es ciudadano paraguayo, nación en la cual se reconocen dos lenguas oficiales (español y guaraní), una de las cuales Achokalla también habla como segunda lengua, ¿es Achokalla, en tanto hablante, paraguayo o aymara?

Como se observa, es una cuestión o conjunto de cuestiones complejas. Un factor, no obstante, se nos presenta nítidamente: cuando hablamos de la propiedad de una lengua nos estamos refiriendo a la antigua noción jurídica occidental de herencia. Originaria del pensamiento griego, es en la tradición jurídica romana que se establece sólidamente, para definir al acto de transmisión de bienes, derechos y obligaciones de una persona física o jurídica a otra. En estos términos estrictos, el término herencia (latín: hereditas, -atis) sirvió para asegurar la permanencia de la familia troncal y su propiedad, no siempre material, a lo largo de las generaciones, o sea la sucesión (latín: successio, -onis), término que debe entenderse también como descendencia o progenie. En sus orígenes, la herencia se vinculaba a lo colectivo y a asegurar su continuidad. En los tiempos posteriores adquirió en el derecho occidental un valor inter-individual, a medida que la propiedad se descolectivizó, pero su uso también se extendió a otros dominios con el sentido original colectivista, como por ejemplo el biológico (la herencia genética, es decir la transmisión de los caracteres fisiológicos, morfológicos y bioquímicos de los seres vivos), el social (herencia moral, referida a los valores y rasgos de personalidad atribuibles a un grupo), hasta llegar al cultural (herencia cultural, o sea el patrimonio cultural de una comunidad).

El patrimonio cultural es la herencia proveniente del pasado de una comunidad en términos acumulativos y de vigencia, es decir: lo que ésta heredó, vive en la actualidad y transmitirá a las generaciones futuras. En su concepción estándar, el patrimonio cultural se distingue de la simple paternidad (la descendencia a partir de padres comunes en la sucesión lineal) y no se entiende como herencia muerta, sino a partir de lo que las generaciones reconozcan como parte integrante de su patrimonio cultural, recreándolo constantemente en función de su historia, su entorno y sus vínculos con otras comunidades, de lo cual se derivan lealtades, identificaciones y continuidad teleológica. El patrimonio cultural incluye como bien tangible o intangible, según el alcance que se les dé a estos adjetivos, a la lengua, casi siempre en una jerarquía de destaque, que en algunas composiciones teóricas se entiende como bien patrimonial esencial y principal. 
En el marco de referencia de las investigaciones acerca de las comunidades hispanas en contacto con las anglosajonas en los Estados Unidos, y sobre todo en relación con las opciones educativas y de enseñanza en contextos bilingües, Valdez (1995; 2005) desarrolló el concepto de lengua de herencia (inglés: heritage language). Originalmente restricto a estos espacios y márgenes operativos, el concepto se refiere más bien a los hablantes que a las lenguas en sí, como aquellos que hablan o comprenden una variedad lingüística en su ámbito de pertenencia (hogar, microespacio social, etc.) que no es la lengua oficial del lugar en que viven. La enseñanza en/de esa lengua de herencia, que muchas veces se ha instrumentado como enseñanza de lengua extranjera (o de segunda lengua) debe seguir, según Valdez otros derroteros.

En su uso más general, el término lenguas de herencia ha sido utilizado para definir a todas aquellas lenguas que conviven con otras lenguas dominantes en contextos bilingües o multilingües, generalmente constituidas por lenguas indígenas o lenguas habladas por grupos de inmigrantes. Particularmente en los Estados Unidos, este concepto ha tenido amplia utilización y las investigaciones que ha permitido son ya un campo específico, como lo muestra la existencia y pujanza del National Heritage Language Resource Center (NHLRC, s/f). A nivel internacional, el concepto se ha difundido mucho, pero no alcanza a ser jerarquizado de la misma manera. Se trata, como es obvio, de todas aquellas lenguas habladas y conservadas por grupos lingüísticos minoritarios ${ }^{9}$, aunque en muchos casos se ha pasado por alto esta particularidad. En los pliegues de la ideología estadounidense dominante, y en el contexto de una visión instrumentalista del lenguaje que jerarquiza las "acciones" de los hablantes por sobre las determinaciones históricas, es posible leer en los trabajos referidos a estas posiciones interpretativas y analíticas (e. g. FISHMAN, 2001; PASCUAL y CABO/ROTHMAN, 2012; POLINSKY/KAGAN, 2007) una serie de contradicciones y aporías que consideramos a continuación, sin que podamos desarrollar nuestro análisis con la extensión debida.

No debería pasarse por alto que toda lengua es "de herencia", y que los rasgos intrínsecos de herencia que la definen derivan de su historicidad recreada en cada acto de habla y de las particularidades del patrimonio cultural del grupo del cual proviene, no de las posiciones que les confieren los hablantes como actores independientes. Teóricamente no hay substancia para definir a unas lenguas como de herencia en relación a otras que no lo sean; en todo caso podría decirse que unas lenguas son usadas en ciertos sistemas educativos como "domésticas" o "de pertenencia" en oposición a lenguas oficialmente reconocidas como "nacionales" (y quizá a otras de origen "extranjero" o de presencia "secundaria").

El concepto de lengua de herencia remite a la condición de hablante, es decir a las relaciones de los sujetos con las lenguas. La relación-materna o condición-materna no define una lengua por su carácter intrínseco, como en expresiones del tipo "lengua alemana", "lengua aglutinante" o "lengua minoritaria", sino por el tipo de relación que la lengua en cuestión establece con un cuerpo que vendrá a ser su hablante (MILNER, 1978). Cierta ilogicidad parece separar en estos análisis la condición materna de la lengua de herencia, con base en la definición muy cuestionable de que "lengua materna es aquella que mejor se habla", y la constatación de que en muchos casos ese mejor dominio se da en segundas y terceras generaciones en la lengua de la comunidad plural que ha sometido a la lengua minoritaria 
La fuerte tendencia de este tipo de estudios hacia una visión de "contacto" o de integración de herencias y grupos minoritarios, sobre la base de una cultura general de tipo nacional, enmascara el carácter de conflicto político en que se asienta la relación entre los grupos lingüísticos. En cierta forma, el término herencia es usado aquí con un tono de minoridad e imperfección, que reproduce los conflictos de poder entre la comunidad angloparlante y las que vinieron a insertarse en su seno o las que ocupaban un territorio antes de la llegada de los angloparlantes ${ }^{10}$. En otras realidades geopolíticas, este enmascaramiento se sostiene, en general, por el olvido de la condición material etnolingüística de los grupos y por la interpretación de su existencia en términos folklóricos más o menos superficiales.

\section{Comentario final}

Nuestro análisis, a pesar de su brevedad y carácter abstracto y sintético, nos permite llamar la atención sobre los problemas teóricos y jurídicos implicados en la instrumentación de los principios de inclusión social y cultural y de reconocimiento de derechos lingüísticos en los ámbitos de la educación. Específicamente, pretendemos haber aportado una discusión preliminar acerca de la operatividad, consistencia teórica y justicia de las tendencias basadas en las modalidades de instrumentación conocidas como tolerancia, promoción y herencia, características del pensamiento liberal y de las concepciones del lenguaje y las lenguas en términos de uso individual.

Hay, también, una amplia casuística que fue necesario considerar para constituir nuestro análisis; su descripción e interpretación, por razones de espacio y oportunidad, deberá no obstante ser postergada para futuras etapas de nuestra indagación.

\section{Referencias}

BEHARES, L. E. Notas sobre la noción de Derechos Lingüísticos ¿Quién es su titular? RDPL -Revista Digital de Políticas Lingüísticas, Año 4, v. 4. p. 37-58. 2013 a.

BEHARES, L. E. Hablantes y Comunidades: Crítica de la noción estándar de Derechos Lingǘsticos. In: FARENZENA, N. (Org.) VI Encontro Internacional de Investigadores de Políticas Lingüísticas. Porto Alegre: Universidade Federal de Rio Grande do Sul - Associação de Universidades Grupo Montevideo, p. 33-41.2013b.

CAPOTORTI, F. Study on the Rights of Persons Belonging to Ethnic, Religious and Linguistic Minorities. New York: United Nations. 1979.

DYKE, V. van The individual, the state, and ethnic communities in political theory. World Politics, 29: 343-369.1977.

CMCE-Comité de Ministros del Consejo de Europa. Carta Europea de las lenguas regionales y minoritarias, labrada en Estrasburgo el 5 de noviembre de 1992. www.consello.org/pdf/ cartaeuropea92.pdf (consultada 20/01/13).

FISHMAN, J. A. Reversing Language Shift: theoretical and empirical foundations of assistance to threatened languages. Clevedon: Multilingual Matters. 1991.

FISHMAN, J. A. 300-plus years of heritage language education in the United States. In PEYTON, J. K.; RANARD, D. A.; MCGINNIS, S. (Eds.) Heritage Languages in America: Preserving a National Resource. Washington, DC: Delta Systems Co. and Center for Applied Linguistics, p. 81-98. 2001. 
FRANCIA. Déclaration des droits de l'homme et du citoyen. In: COLLIARD, C-A. La déclaration des droits de l'homme et du citoyen de 1789. Paris: La documentation française, 1990, p. 15-19. (fecha original: 1789).

HOLBOROW, M. The Politics of English: a Marxist view of language. London: Sage. 1999.

INGLATERRA Habeas Corpus Amendment Act (26 de marzo de 1679). In: Biblioteca Jurídica Virtual Del Instituto de Investigaciones Jurídicas. México: Universidad Autónoma de México. www.juridicos.unam.mx, p. 185-193. (Consultada 20/01/14) (fecha original: 1679)

INGLATERRA Bill of Rights (1689). In: Biblioteca Virtual Miguel de Cervantes. Madrid, www.cervantesvirtual.com/obra/bill-of-rights-1689--0 (Consultada 20/01/14) (Fecha original: 1689).

KLOSS, H. The American Bilingual Tradition. Rowley: Newbury House. 1977.

KYMLICKA, W. Multicultural Citizenship: a liberal theory of minority rights. Oxford: Clarendon Press. 1995.

MAY, S. Derechos lingüísticos como derechos humanos. Revista de Antropología Social, 19, 131-159. 2010.

MILNER, J- C. L'Amour de la Langue. Paris: Du Seuil. 1978.

NHLRC - National Heritage Language Resource Center. (Portal). Acceso en: www.nhlrc.ucla. edu (Consultada em 10/02/14). s/f.

NIC CRAITH, M. Europe and the Politics of Language: Citizens, migrants, and outsiders. London: Palgrave-Macmillan. 2006.

ONU- Organización de las Naciones Unidas. Carta de las Naciones Unidas. Sitio Institucional de ONU, www.un.org/es/documents/charter/ (consultada 20/01/13). (Fecha original: 1945).

ONU- Organización de las Naciones Unidas. Declaración Universal de los Derechos Humanos. www.un.org/es/documents/udhr (consultada 20/01/13). (Fecha original: 1948)

ONU- Organización de las Naciones Unidas. Pacto Internacional de Derechos Civiles y Políticos. Página de la Oficina del Alto Comisionado de las Naciones Unidas para los Derechos Humanos. www2 .ohchr.org/spanish/law/ccpr.htm (consultada 20/01/13). (Fecha original: 1966)

ONU- Organización de las Naciones Unidas. Proclamación de Teherán. http://www.gloobal. net/iepala/gloobal/fichas/ficha.php?entidad=Textos\&id=4052 (consultada 20/01/13). (Fecha original: 1968)

ONU- Organización de las Naciones Unidas. Convención sobre los Derechos del Niño. Página oficial de UNICEF, www.unicef.org/.../spanish/7.-Convencionsobrelosderechos.pdf (consultada 20/01/13). (Fecha original: 1989)

ONU- Organización de las Naciones Unidas. Declaración sobre los derechos de las personas pertenecientes a minorías nacionales o étnicas, religiosas y lingüísticas. Página de la Oficina del Alto Comisionado de las Naciones Unidas para los Derechos Humanos. www2.ohchr.org/ spanish/law/minorias.htm (consultada 20/01/13). (Fecha original: 1992).

PASCUAL y CABO, D.; ROTHMAN, J. The (il)logical problem of heritage speaker bilingualism and incomplete acquisition. Applied Linguistics 33 (4): p. 450-455. 2012.

POLINSKY, M.; KAGAN, O. Heritage languages: In the "wild" and in the classroom. Language and Linguistics Compass 1 (5): p. 368-395. 2007.

SKUTNABB-KANGAS, T. Human rights and language wrongs - a future for diversity? Language Sciences, 20: 5-27. 1998. 
UNESCO- Organización de las Naciones Unidas para la Educación, la Ciencia y la Cultura. Empleo de las Lenguas Vernáculas en la Enseñanza. París: Ediciones de la Unesco. 1953.

UNESCO- Organización de las Naciones Unidas para la Educación, la Ciencia y la Cultura Convención de la UNESCO relativa a la recomendación sobre la lucha contra las discriminaciones en la esfera de la enseñanza. Portal oficial de UNESCO. portal.unesco.org/es/ev.php -URL_ID=12949\&URL_DO=DO_TOPIC\&... (consultada 20/01/13). (Fecha original: 1960)

UNESCO. Organización de las Naciones Unidas para la Educación, la Ciencia y la Cultura. Declaración Universal de los Derechos Lingüísticos. Página Oficial de UNESCO. http:// www.unesco.org/cpp/sp/declaraciones/linguisticos.htm (consultada 20/01/13). (Fecha original: 1996).

VALDEZ, G. The teaching of minority languages as "foreign" languages: Pedagogical and theoretical challenges. Modern Languages Journal, v. 79, núm. 3, p. 299-328. 1995.

VALDEZ, G. Bilingualism, heritage language learners, and SLA research: Opportunities lost or seized? The Modern Language Journal 89 (3): p. 410-426. 2005.

VARENNES, F. de. Language, Minorities and Human Rights. The Hague: Kluwer Law International. 1996.

YOUNG, I. Together in Difference: transforming the logic of group political conflict. In: SQUIRES, J. (Ed.) Principled Positions: postmodernism and the rediscovery of value. London: Lawrence and Wishart: pp. 121-150.1993.

\section{Notas}

${ }^{1}$ Producto de la Línea de Investigación "Dimensiones Lenguajeras de la Enseñanza y el Aprendizaje" (DLEyA), llevada adelante en el Departamento de Enseñanza y Aprendizaje (Instituto de Educación, Universidad de la República, Montevideo-Uruguay).

2 Entre otros, los documentos principales son: CMCE (1992); ONU (1945, 1948, 1966, 1968, 1989, 1992); UNESCO $(1953,1960,1996)$.

${ }^{3}$ Una cuestión técnica: la noción contemporánea de "derechos lingüísticos" fue generada en el ámbito de la legislación internacional ligada a los derechos humanos o "humanitarios" (ONU, 1945, art. 68; ONU, 1948), en primera instancia, y luego comenzó a ser tomada como materia de las legislaciones positivas nacionales.

${ }^{4}$ Es éste el espíritu de los documentos fundadores de esta conceptualidad, como el Habeas Corpus Act de 1679 (Inglaterra, 1679) y el Bill of Rights de 1689 (Inglaterra, 1689) y de la La déclaration des droits de l'homme et du citoyen francesa de 1789 (Francia 1789).

${ }^{5}$ e. g. ONU (1989, arts. 17, 20 y 30); ONU (1992, arts. 1 y 4).

${ }^{6}$ Este documento fue aprobado en la Conferencia Mundial de Derechos Lingüísticos de Barcelona, organizada por varias instituciones y asociaciones con el apoyo "moral y técnico" de UNESCO. No es un documento preceptivo para los países, pero se lo ha jerarquizado como una summa en la materia.

${ }^{7}$ Se pueden encontrar, obviamente, arreglos más relativistas, como los de Kymlicka (1995) en su noción de “derechos diferenciados", básicamente en la afirmación de que los grupos permiten algunas variaciones propositivas para derechos esencialmente individuales afectados por las construcciones culturales o históricas.

${ }^{8}$ Nic Craith (2006) encuentra similares problemas en la Carta Europea (CMCE, 1992) y en otros documentos posteriores. 


\section{Luis Ernesto Behares}

${ }^{9}$ Minoritarios, claro está, no sólo en el aspecto demográfico cuantitativo, sino también, y a veces principalmente, en sus condiciones de poder (social, político, económico, cultural).

${ }^{10}$ Es muy interesante comprobar que en la mayoría de estas composiciones teóricas se remite como realidad empírica paradigmática a la de los llamados "hispanos" o "latinos", expresiones cuyo único sentido deriva de no ser parte de las comunidades angloparlantes, ya que los factores étnico-culturales asociados y la propia naturaleza de las variedades lingüísticas que hablan son extremadamente disímiles.

* Profesor Titular del Departamento de Enseñanza y Aprendizaje, Instituto de Educación, Universidad de la República, Montevideo - Uruguay.

\section{Correspondência}

Luis Ernesto Behares - Instituto de Educación, Facultad de Humanidades y Ciencias de la Educación, Magallanes 1577, 11200 Montevideo, Uruguay.

E-mail:1beharesc@yahoo.com

Recebido em 04 de janeiro de 2014

Aprovado em 30 de março de 2014 